\title{
Immediate Limb Compression Following Supermicrosurgical Lymphaticovenular Anastomosis - Is It Helpful or Harmful?
}

\author{
Wei F. Chen, MD, FACS*; Mindy Bowen, RN; Johnson Ding, BS \\ Division of Plastic and Reconstructive Surgery, Department of Surgery, \\ University of lowa Hospitals and Clinics, lowa City, lowa, USA
}

\begin{abstract}
Background: Lymphaticovenular anastomosis (LVA) is an established supermicrosurgical treatment of the lymphedema. However, success rates vary, possibly related to the variation in patient selection, surgical technique, and postoperative care. One of the controversies on postoperative care is whether to apply limb compression. We set out to assess the effect of external limb compression on the LVA.

Methods: Following each of the anastomoses of the LVA procedure, the flow across the anastomosis was immediately assessed. A "washout" sign was defined as observing the favorable ante grade, lymph-to-vein flow, whereas a "backflow" sign was defined as observing the unfavorable retrograde, vein-to-lymph flow. After the initial flow pattern was recorded, bandage compression was applied to the leg and the changes to the flow pattern were recorded. Patients were tracked with lymphedema indices as well as lymphedema quality of life (LYMQOL) assessment system at preoperative, within the 3rd and 6th month visits.

Results: Five patients were included in the study. 42 LVAs were constructed - 26 with the standard, and 16 via the octopus technique. Initially, 25 (60\%) demonstrated "washout", with the remaining 17 (40\%) showing "backflow". After compression was applied, those entire initially demonstrating washout" maintained the "washout" pattern, while 16 of 17, or $94 \%$, that initially demonstrated "backflow" converted to "washout". In the follow up, all patients had statistically significant edema reduction based on lower extremity lymphedema indices $(P=0.0009)$ and relief of symptoms based on the LYMQOL assessment $(P=0.0006)$.

Conclusion: Postoperative compression following LVA does not harm the anastomoses created, and can augment the lymphatic flow and convert unfavorable retrograde flow to favorable ante grade flow.
\end{abstract}

\section{Introduction}

Lymphaticovenular anastomosis (LVA) is a minimally invasive, supermicrosurgical alternative to vascularized lymph node transfer (VLNT) for lymphedema reconstruction [1-3]. The procedure is conceptually simple, and it involves making lymph-to-vein connections via small skin incisions. In contrast to VLNT [4,5], the procedure does not involve harvesting lymphatic tissue and is therefore free of the risk of causing donor-site lymphedema. While its minimally invasive nature is appealing, its reported outcome is disappointingly inconsistent [6-8]. The inconsistency in outcome is likely related to the differences in surgical technique and peri-operative care. The application of postoperative limb compression is one of these controversial differences. Intuitively, limb compression following the LVA procedure may mechanically narrow these minuscule anastomoses, causing anastomotic failure. In standard microsurgery, pressure avoidance at the anastomotic site is an unchallenged dictum. But does the same hold true for the supermicrosurgical LVA? We conducted a simulation study to answer this question does limb compression following LVA promote or impair lymph-to-vein drainage?

\section{Method}

\section{Patients}

Five consecutive patients, four female and one male, with age ranging from 23 years to 69 years; undergoing LVA for limb lymphedema, were included in the study (Table 1). All patients had previously failed complex decongestive lymphedema therapy and were referred by our lymphedema therapists for evaluation for surgical reconstruction. All had lower extremity lymphedema. Three had acquired disease and two had primary disease. The severity of disease was staged with Campisi criteria and all had stage II and III diseases.

\section{Table 1. Comparison of Pre-operative and Post-operative LEL Indices and LYMQOL Scores}

\begin{tabular}{|c|c|c|c|c|c|c|c|}
\hline Patient & Age & Extremity & Etiology & Campisi Stage & No. of LVA & LEL Indices (Pre/Post) & LYMQOL (Pre/Post) \\
\hline 1 & 52 & Leg & Acquired & II & 10 & $294 / 279$ & $82 / 57$ \\
\hline 2 & 69 & Leg & Acquired & II & 8 & $312 / 294$ & $97 / 62$ \\
\hline 3 & 23 & Leg & Congenital & III & 9 & $337 / 312$ & $103 / 79$ \\
\hline 4 & 58 & Leg & Congenital & II & 7 & $298 / 282$ & $78 / 46$ \\
\hline 5 & 67 & Leg & Acquired & III & 8 & $319 / 305$ & $94 / 53$ \\
\hline
\end{tabular}

Lymphedema index is a circumference-based system that takes measurements at five limb levels and references the sum to the patient's body mass index. LYMQOL is a validated lymphedema-specific quality of life assessment that tracks four condition-specific domains - function, appearance, symptoms, and mood. LEL, lower extremity lymphedema; LYMQOL, lymphedema-specific quality of life assessment; LVA, lymphaticovenular anastomosis; Pre, pre-operative; Post, post-operative. 

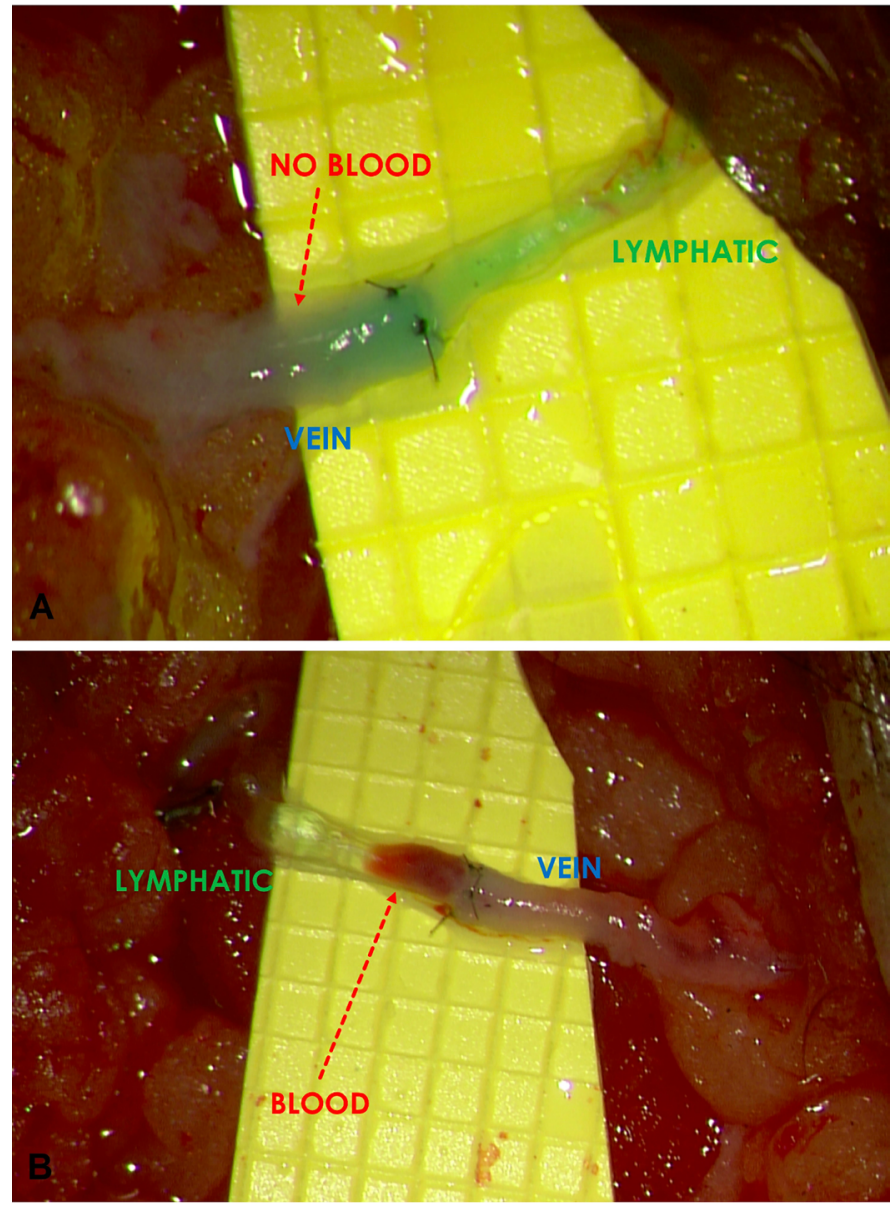

Figure 1. (A) A favorable "washout" sign was observed when the lymphatic pressure exceeded the venous pressure and ante grade flow occurred. (B) Conversely, retrograde flow occurred when the venous pressure exceeded the lymphatic pressure and an unfavorable "backflow" sign was shown.

\section{Study Design}

Intraoperatively, immediately following the completion of each of the individual LVAs, the flow pattern across the anastomosis was observed under the surgical microscope. When the lymphatic pressure exceeded the venous pressure, favorable ante grade lymph-to-vein flow occurred and a "washout" sign (Figure 1A) was observed. Conversely, when the pressure gradient was reversed, with the venous pressure exceeding the lymphatic pressure, unfavorable retrograde flow occurred and a "backflow" sign was seen (Figure 1B). Regardless of the initial flow pattern observed, bandage compression was applied to the entire limb to simulate postoperative limb compression (Figure 2). The firmness of compression was determined by the senior author to simulate the compression pressure of $30-40 \mathrm{mmHg}$. Changes to the flow pattern following the bandage compression were observed under the microscope and recorded. Patient evaluation was performed using the circumference-based lower extremity lymphedema (LEL) index system [9] and a lymphedema-specific quality of life assessment (LYMQOL) [10] at preoperative visit; and at the 3rd and 6thmonth postoperative visits. LYMQOL is a condition-specific validated assessment system that tracks postoperative changes in function, appearance, symptoms, and mood. The preoperative and 6-month postoperative $L E L$ and values were compared using paired t-test.

\section{Surgical Technique}

After mapping the lymphatic vessels with indocyanine green lymphography and delineating the superficial venules with an infrared imaging device (VueTek Scientific, Gray, Maine), the incisions were strategically

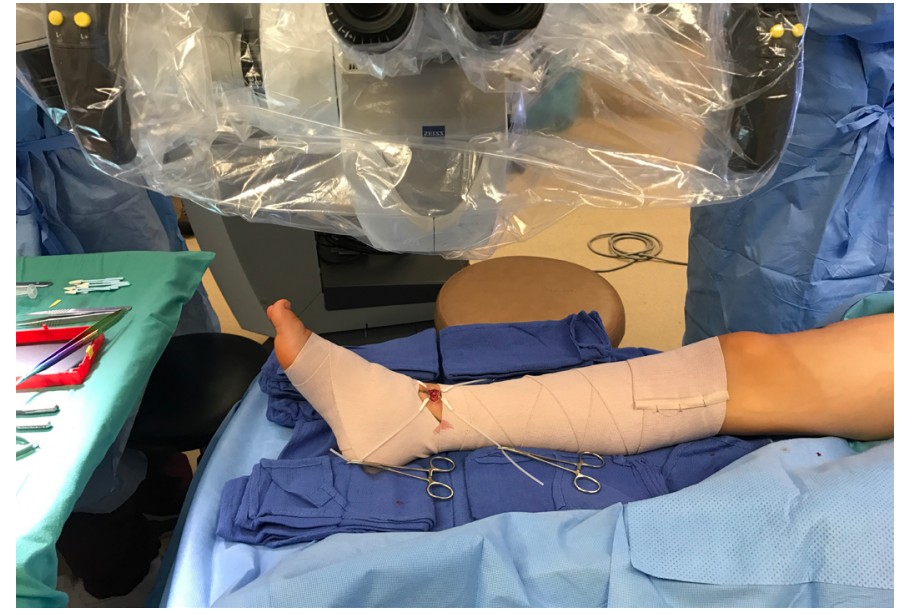

Figure 2. After having documented the initial LVA flow pattern, bandage compression was applied proximal and distal to the incision to simulate the effect of immediate postoperative limb compression.

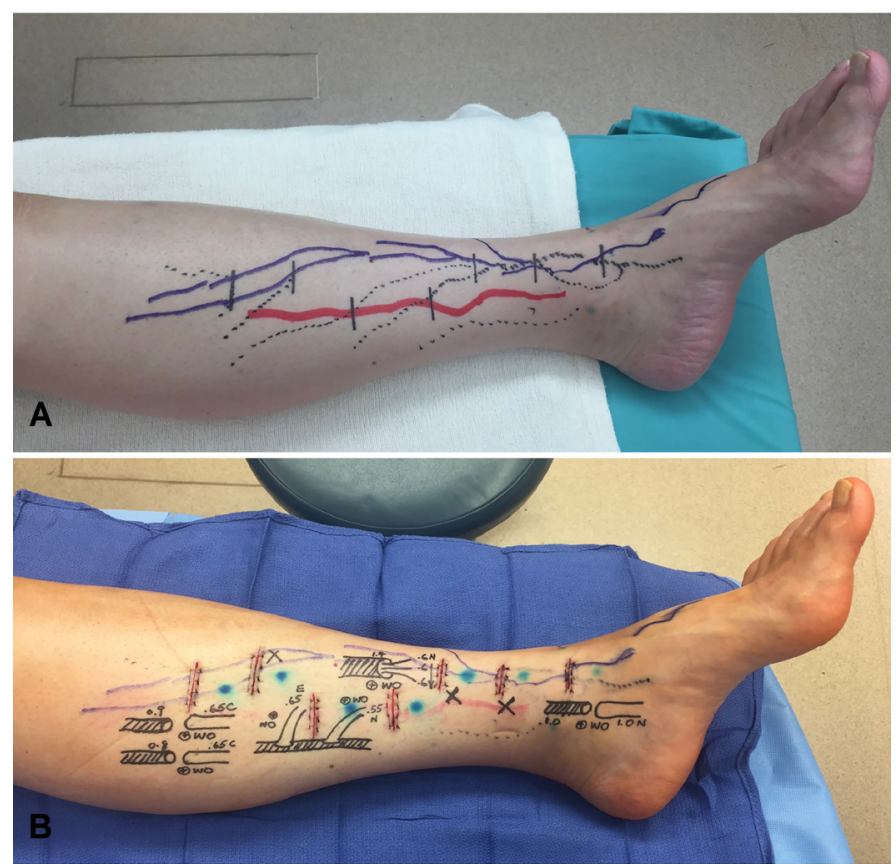

Figure 3. (A) Solid blue and red lines represented lymphatic vessels mapped with indocyanine green lymphography from different injection sites. Dotted lines were superficial venules visualized using the infrared vein finder. Incisions were planned at where the lymphatic vessels and the superficial venules intersected. (B) Postoperative skin markings showing LVAs constructed with various anastomotic techniques.

placed at locations where both the lymphatic vessels and venules were present as previously described [8] (Figure 3). $0.05 \mathrm{cc}$ of isofulfan blue (Lymphazurin; United States Surgical Corp., Norwalk, Connecticut) was injected within $2 \mathrm{~cm}$ distal to each incision to further facilitate identification of the lymphatic vessels. The LVAs were performed at 25X magnification utilizing a surgical microscope (Pentero 900; Carl Zeiss, Oberkochen, Germany) using specialized supermicrosurgical instruments (EMI Factory, Kitasakugun, Nagano, Japan). Both the standard supermicrosurgical LVA technique described by Koshima and Yamamoto et al. [11-13] and the "octopus" technique [14] were used. When healthy lymphatic vessels and size-matched veins were present, the standard technique was pref- 

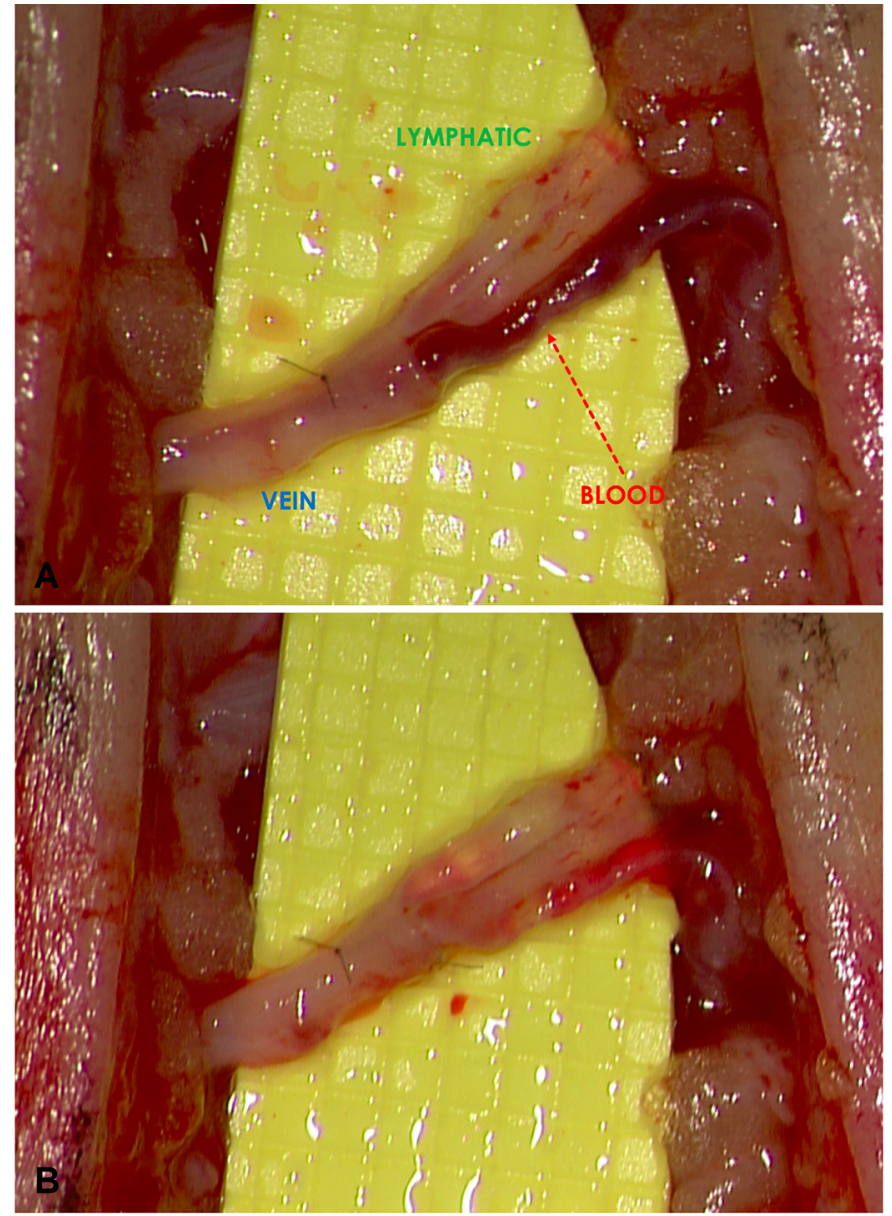

Figure 4. (A) Before compression. A 3 lymphatic vessel-to-1 vein LVA anastomosed using the "octopus" technique showing "backflow" in all 3 lymphatic vessels. The "backflow" sign was particularly prominent in the most inferior lymphatic vessel. (B) After compression. Conversion to "washout" was seen in all 3 of the lymphatic vessels. Note how the blood was "washed out" of all 3 of the lymphatic vessels and the vein. Engorgement of the lymphatic vessels was clearly seen in the top 2 lymphatic vessels.

erentially used. The "octopus" technique was reserved for the challenging situations of 1) lymphatic vessels being severely damaged due to the disease process and 2) number-and/or caliber-mismatch between the lymphatic vessels and the veins. The anastomoses were performed using 12-0 nylon with 50-micrometer needle (S\&T, Neuhausen, Switzerland).

\section{Postoperative Care}

Limb compression was applied immediately following the surgery using the short-stretch bandage. All patients were discharged to home on postoperative day one. Bandage compression continued for 16 hours per day until six weeks postoperatively. At that time, all were transitioned to $30-$ $40 \mathrm{mmHg}$ pressure. Throughout the 6-month study period, all patients continued to wear their pressure garments for 16 hours per day. Weaning of the pressure garments began at 7 months from the surgery and was not evaluated in this study.

\section{Results}

A total of 42 LVAs were created - 26 standard LVAs and 16 "octopus" LVAs. Initially, 25 of the LVAs (60\%) demonstrated the favorable "washout" sign and the remaining 17 (40\%) demonstrated the unfavorable "backflow" sign. Among these 17 LVAs demonstrating the "backflow" sign, 13 were the "octopus" and 4 were the standard LVAs. Following the application of limb compression, 16 of the 17 LVAs (94\%) converted the flow pattern from the unfavorable "backflow" to the favorable "washout" (Figures 4A \& 4B). The conversion rates of the "octopus" and the standard LVAs were $100 \%$ (13/13) and 75\% (3/4), respectively. All 25 LVAs initially demonstrating "washout" maintained the "washout" pattern following the bandage compression. Regardless of the initial flow patterns, post-compression vessel engorgement was observed in the lymphatic vessels (Figure 4B). This finding along with the post-compression flow reversal suggested a compression-induced augmentation of the lymphatic pressure.

All patients demonstrated reduction of limb swelling and relief of symptoms, as demonstrated by statistically significant improvements in the lower extremity lymphedema indices $(P=0.0009)$ and in the LYMQOL $(P=0.0006)$ (Table 1). All patients reported their symptoms being notably more responsive to compression. No patient had worsening of lymphedema symptoms during the 6-month study period.

\section{Discussion}

LVA is a delicate supermicrosurgical procedure. Until now, most studies focused on the intricate technical aspects of the procedure [13-16] and little had been described about postoperative management. As we become more proficient in creating these tiny anastomoses, it is important to start to evaluate other procedural parameters to maximize surgical efficacy. The opinions and practices on postoperative limb compression following LVA vary widely among the supermicrosurgeons. Common practices include no compression [17,18], delayed compression starting few weeks following the surgery [11], and immediate compression [6]. To our knowledge, this is the first simulation study directly evaluating the effects of compression on the LVA.

The findings in this study support immediate postoperative limb compression. When the compression was applied, majority of the unfavorable LVAs with retrograde flow converted to the favorable functioning LVAs with ante grade flow (94\%). When left untreated, the refluxed blood in the lymphatic lumen may cause thrombosis and result in anastomotic failure. Interestingly, the limb compression appeared to create a generalized augmentation of the lymph-to-vein pressure gradient, as suggested by visible engorgement of lymphatic vessels (Figure $4 B$ ). This phenomenon was seen in all the LVAs, including those already demonstrating ante grade flows prior to compression. This finding along with the high rate of favorable flow conversion suggested functional enhancement of the LVAs with postoperative limb compression.

Why would limb compression, which pressurized both the venous and lymphatic systems simultaneously, alter the lymph-vein pressure

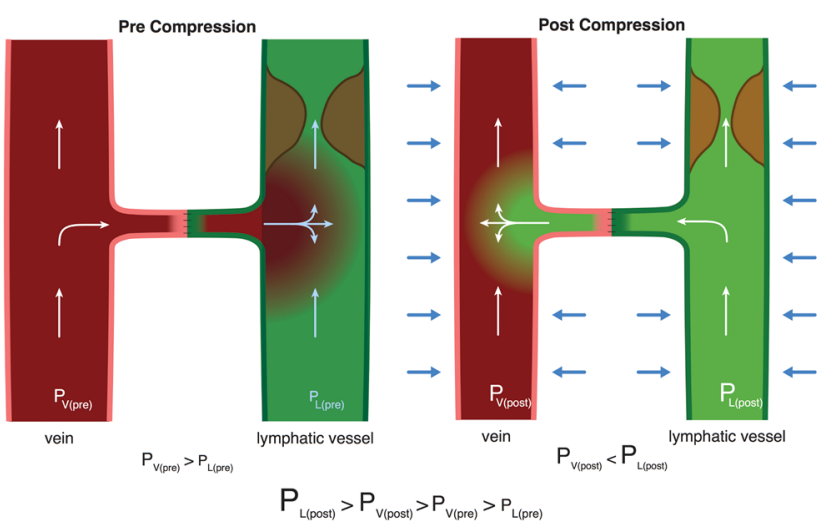

Figure 5. The mechanism of differential pressure changes in the lymphatic and venous systems in response to limb compression. Prior to compression, venous pressure > lymphatic pressure and "backflow" occurred. With compression, the lymphatic system experienced a significantly greater pressure increase due to the presence of outflow obstruction associated with lymphedema, resulting in a reversal of flow and a "washout" sign. 
gradient? We hypothesized that the differential effects on the two systems in response to external pressure may be related to the lymphatic system being a partially obstructed system and the venous system being a non-obstructed, free-flowing system. When both systems were simultaneously pressurized, the partially obstructed system experienced a higher magnitude of pressure increase relative to the open system due to its inability to efficiently decompress (Hai Fu, Department of Physics and Astronomy, University of lowa, personal communication, December 22, 2016) (Figure 5). In contrast, not having an outflow obstruction, the open venous system could quickly decompress and therefore experienced a lesser pressure increase.

We were not surprised by the higher incidence of "backflow" in the "octopus" LVA ( $81 \%$ or 13 of 16 ) relative to the standard LVA because the lymphatic vessels used in the "octopus" technique were qualitatively worse than the ones used in the standard technique, and were mostly of the "contraction type" $[19,20]$. Without compression, these LVAs would likely not be effective due to their inability to peristalze and generate a favorable lymph-to-vein pressure gradient. Using compression, we were able to convert 100\% (13 of 13) of the unfavorable "octopus" LVAs with "backflow" to the favorable, functioning LVA showing "washout". This finding is encouraging because it suggested that even the damaged "contraction type" lymphatic vessels may be successfully recruited to build functioning LVAs. This allows less restrictive lymphatic vessel recruitment and will lead to increased number of LVAs created per surgery. Furthermore, this means that instead of limiting the LVA procedure only to patients with early diseases $[6,21]$, surgery may be considered even in those with intermediate disease severity who tend to have fewer healthy lymphatic vessels.

In summary, the benefits of immediate compression following the LVA are three-fold:1) it converts nonfunctioning LVAs with retrograde flow to functioning ones with ante grade flow, 2) it augments the flow of the functioning LVAs already demonstrating ante grade flow, and 3) it decreases the restrictive nature of the LVA procedure and allows the surgeon to use moderately disease-affected lymphatic vessels, creating higher numbers of functioning LVAs. Currently, the endpoint of limb compression and the timing of its discontinuation are unknown, and they are being investigated in our ongoing studies.

\section{Conclusion}

Immediate limb compression following the LVA procedure facilitates lymphatic drainage and increases the surgical efficacy by increasing the number of functioning anastomoses, and is a recommended postoperative practice.

\section{Article Information}

*Correspondence: Wei F. Chen, MD, FACS

Division of Plastic and Reconstructive Surgery, Department of Surgery, University of lowa Hospitals and Clinics, 20 Hawkins Drive, 1537 JCP, lowa City, lowa, 52242, USA. Email: wei-chen@uiowa.edu

Received: Apr. 30, 2017; Accepted: Oct. 13, 2017; Published: May 23, 2018

DOI: $10.24983 /$ scitemed.imj.2018.00063

Copyright $@ 2018$ The Author (s). This is an open-access article distributed under the terms of the Creative Commons Attribution 4.0 International License (CC-BY).

\section{Funding: None}

\section{Conflict of Interest: None}

\section{Keywords}

Lymphedema; lymphaticovenular anastomosis; lymphedema surgery; supermicrosurgery.

\section{References}

1. Yamamoto $T$, Narushima $M$, Yoshimatsu $H$, et al. Minimally invasive lymphatic supermicrosurgery (mils): indocyanine green lymphography-guided simultaneous multisite lymphaticovenular anastomoses via millimeter skin incisions. Ann Plast Surg 2014;72(1):67-70.

2. Cheng $\mathrm{MH}$, Huang JJ, Nguyen $\mathrm{DH}$, et al. A novel approach to the treatment of lower extremity lymphedema by transferring a vascularized submental lymph node flap to the ankle. Gynecol Oncol 2012; 126(1):93-98

3. Cheng MH, Chen SC, Henry SL, Tan BK, Lin MC, Huang JJ. Vascularized groin lymph node flap transfer for postmastectomy upper limb lymphedema: flap anatomy, recipient sites, and outcomes. Plast Reconstr Surg 2013;131(6):1286-1298.

4. Lin $\mathrm{CH}$, Ali R, Chen SC, et al. Vascularized groin lymph node transfer using the wrist as a recipient site for management of postmastectomy upper extremity lymphedema. Plast Reconstr Surg 2009;123(4):1265-1275.

5. Cheng $\mathrm{MH}$, Huang JJ, Wu CW, et al. The mechanism of vascularized lymph node transfer for lymphedema: natural lymphaticovenous drainage. Plast Reconstr Surg 2014;133(2):192e-198e.

6. Chang DW, Suami H, Skoracki R. A prospective analysis of 100 consecutive lymphovenous bypass cases for treatment of extremity lymphedema. Plast Reconstr Surg 2013;132(5):1305-1314.

7. Damstra RJ, Voesten HGJ, van Schelven WD, van der Lei B. Lymphatic venous anastomosis (LVA) for treatment of secondary arm lymphedema. A prospective study of 11 LVA procedures in 10 patients with breast cancer related lymphedema and a critical review of the literature. Breast Cancer Res Treat 2009;113(2): 199-206.

8. Chen WF, Zhao H, Yamamoto T, Hara H, Ding J. Indocyanine Green Lymphographic Evidence of Surgical Efficacy Following Microsurgical and Supermicrosurgical Lymphedema Reconstructions. J Reconstr Microsurg 2016;32(9): 688-698.

9. Yamamoto T, Matsuda N, Todokoro T, et al. Lower Extremity Lymphedema Index: a simple method for severity evaluation of lower extremity lymphedema. Ann Plast Surg 2011;67(6):637-640.

10. Keeley V, Crooks S, Locke J, Veigas D, Riches K, Hilliam R. A quality of life measure for limb lymphoedema (LYMQOL). Journal of Lymphoedema 2010;5:26-37.

11. Koshima I, Inagawa K, Urushibara K, Moriguchi T. Supermicrosurgical lymphaticovenular anastomosis for the treatment of lymphedema in the upper extremities. J Reconstr Microsurg 2000;16(6):437-442.

12. Yamamoto $T$, Yoshimatsu $H$, Narushima $M$, et al. A modified side-toend lymphaticovenular anastomosis. Microsurgery 2012;33(2):130133.

13. Yamamoto T, Chen WF, Yamamoto N, Yoshimatsu H, Tashiro K, Koshima I. Technical simplification of the supermicrosurgical sideto-end lymphaticovenular anastomosis using the parachute technique. Microsurgery 2015;35(2):129-134.

14. Chen WF, Yamamoto T, Fisher M, Liao J, Carr J. The "Octopus" Lymphaticovenular anastomosis: evolving beyond the standard supermicrosurgical technique. J Reconstr Microsurg 2015;31(6):450-457.

15. Yamamoto T, Narushima M, Kikuchi K, et al. Lambda-shaped anastomosis with intravascular stenting method for safe and effective lymphaticovenular anastomosis. Plast Reconstr Surg 2011;127(5):19871992.

16. Ayestaray B, Bekara F. $\pi$-shaped lymphaticovenular anastomosis: the venous flow sparing technique for the treatment of peripheral lymphedema. J Reconstr Microsurg 2014;30(8):551-560.

17. Auba C, Marre D, Rodríguez-Losada G, Hontanilla B. Lymphaticovenular anastomoses for lymphedema treatment: 18 months postoperative outcomes. Microsurgery 2012;32(4):261-268.

18. Mukenge $S$, Negrini $D$, Catena $M$, et al. Development of functionally patent lymphatic meshes in postsurgical long-term resolution of peripheral secondary lymphedema. J Vasc Surg Venous Lymphat Disord 2013;1(3):280-288. 


\section{ORIGINAL}

19. Mihara $\mathrm{M}, \mathrm{Hara} H$, Hayashi $\mathrm{Y}$, et al. Pathological steps of cancer-related lymphedema: histological changes in the collecting lymphatic vessels after lymphadenectomy. PLoS One 2012;7(7):e41126.

20. Hara H, Mihara M, Seki Y, Todokoro T, lida T, Koshima I. Comparison of indocyanine green lymphographic findings with the conditions of collecting lymphatic vessels of limbs in patients with lymphedema. Plast Reconstr Surg 2013;132(6):1612-1618.

21. Masia J, Pons G, Rodríguez-Bauzà E. Barcelona Lymphedema Algorithm for Surgical Treatment in Breast Cancer-Related Lymphedema. J Reconstr Microsurg 2016;32(5):329-335. 